\title{
Sozialpolitische Erzählungen - Ein Vergleich narrativer Strategien in der Finanzkrise und der Corona-Krise
}

\author{
Johanna Kuhlmann (D) Sonja Blum (D)
}

Angenommen: 12. Januar 2022 / Online publiziert: 7. Februar 2022

(C) Der/die Autor(en) 2022

Zusammenfassung Wohlfahrtsstaaten gleichen ökonomische Ungleichheiten nicht nur aus: Sie schaffen diese auch, indem sie stratifizierend wirken und verschiedenen Bevölkerungsgruppen unterschiedliche soziale Rechte gewähren. Durch Reformen werden neue soziale Rechte geschaffen oder bestehende modifiziert; es werden Gruppen neue soziale Rechte gewährt oder soziale Rechte entzogen. Für die Legitimierung sozialpolitischer Reformen - und damit einhergehend: die Reduzierung oder Schaffung ökonomischer Ungleichheiten - spielen Narrative eine zentrale Rolle. Sie transportieren eine bestimmte und in der Regel vereinfachte Situationsbeschreibung, und zeichnen sich durch spezifische Strukturmerkmale aus. Von zentraler Bedeutung ist, wie die Zielgruppen der sozialpolitischen Reformen in diesen Narrativen konstruiert werden. Insbesondere bauen entsprechende Konstruktionen auf einer Unterscheidung von ,,verdienten“ und „unverdienten“ Gruppen auf, die dementsprechend mit höherer/niedrigerer Wahrscheinlichkeit durch sozialpolitischen Aus- oder Rückbau adressiert werden. Der Beitrag untersucht, wie sozialpolitische Reformen in Zeiten akuter Krisen über Narrative kommuniziert werden, und wie die zentralen Zielgruppen in diesen Narrativen konstruiert werden. Mit der Kurzarbeit und dem Elterngeld werden dabei zwei Policies untersucht, die sowohl in der Finanzkrise 2008/2009 als auch in der Corona-Krise prominent wurden.

Schlüsselwörter Wohlfahrtsstaat $\cdot$ Krise $\cdot$ Zielgruppen · Deservingness · Legitimierung

Dr. Johanna Kuhlmann ( $\varangle)$

SOCIUM Forschungszentrum Ungleichheit und Sozialpolitik, Universität Bremen,

Mary-Somerville-Straße 7, 28359 Bremen, Deutschland

E-Mail: johanna.kuhlmann@uni-bremen.de

Dr. Sonja Blum

Institut für Politikwissenschaft, FernUniversität in Hagen, Universitätsstraße 33, 58084 Hagen,

Deutschland

E-Mail: sonja.blum@fernuni-hagen.de 


\title{
Social policy stories-A comparison of narrative strategies in the financial crisis and the COVID-19 crisis
}

\begin{abstract}
Welfare states not only diminish economic inequalities; they can also create them through their stratifying effects, and by granting different social rights to different groups in society. Through social policy reforms, social rights are granted or withdrawn. For legitimating those social policy reforms-and therewith: for legitimating the reduction or reinforcement of economic inequalities - narratives play a crucial role. They are characterised by portraying the problem situation in a specific, often simplified way, and by certain structural features. It deserves special attention how the target populations of social policy reforms are constructed within those narratives, particularly with regard to the distinction of "deserving" and "undeserving" groups - who according to these (un)deservingness constructions are more or less likely to be affected by social policy expansion versus retrenchment. In this article, we analyse how social policy reforms in times of severe and urgent crisis are communicated through policy narratives, and how these narratives construct the main target groups of the reforms. At the focus of the analysis are two policies, which became prominent in Germany in the aftermath of both the financial crisis 2008/09, and the COVID-19 crisis: short-term work, and the parental-leave benefit.
\end{abstract}

Keywords Welfare state $\cdot$ Crisis $\cdot$ Target groups $\cdot$ Deservingness $\cdot$ Legitimation

\section{Einleitung}

Wohlfahrtsstaaten gleichen ökonomische Ungleichheiten nicht nur aus: Sie schaffen diese auch, indem sie stratifizierend wirken und verschiedenen Bevölkerungsgruppen unterschiedliche soziale Rechte gewähren (Esping-Andersen 1990; Mätzke 2011). Durch Reformen werden neue soziale Rechte geschaffen oder bestehende modifiziert; es werden Gruppen neue soziale Rechte gewährt oder bestehende soziale Rechte entzogen. Im politischen Prozess müssen politische Entscheidungsträgerinnen ${ }^{1}$ solche Reformen gegenüber dem politischen Gegner, den eigenen Wählern und der Öffentlichkeit legitimieren. Dabei haben insbesondere die Hartz-Reformen gezeigt, dass bei unpopulären sozialpolitischen Reformen, die nicht überzeugend kommuniziert werden (können), desaströse politische Auswirkungen möglich sind (z.B. Fervers 2019). So konstatieren auch Gilbert et al. (1993, S. 71): „In the real world few social welfare policy issues engender more vigorous debate than who shall benefit and the manner in which entitlement is defined."

Besonderes Konfliktpotential können solche Debatten in Zeiten umfassender Krisen entfalten. Insbesondere dann, wenn Krisen akute und existentielle Bedrohungen für Bürgerinnen darstellen, besteht eine klare Erwartung an die Politik, Lösungen (schnell) zu entwickeln (Boin et al. 2016). Zwei solche umfassenden Krisen, die (z.B. im Gegensatz zur Klimakrise) mit einem kurzfristigen Handlungsdruck ver-

\footnotetext{
1 Wir verwenden hier abwechselnd und zufällig gestreut das generische Maskulinum bzw. Femininum, wobei Personen anderen Geschlechts stets mitgemeint sind.
} 
bunden wurden, bildeten die Finanzkrise 2008/2009 sowie die Corona-Pandemie ab 2020. Gewendet auf die Sozialpolitik bedeutet dies, dass sozialpolitische Instrumente eingesetzt werden, um Bürger materiell abzusichern oder den Zugang zu sozialen Dienstleistungen zu ermöglichen und somit als „flexibles Instrument der Krisenbewältigung“ (Blank 2020) zu fungieren. Welche Gruppen dabei mit Maßnahmen - wie z.B. Kurzarbeitergeld oder Steuererleichterungen - unterstützt werden und welche nicht, bzw. bei welchen Leistungen und Gruppen ggf. aufgrund gestiegener Ausgaben oder einer verschlechterten wirtschaftlichen Situation auch gespart wird, kann nicht nur im politischen Diskurs zu einer Existenzfrage werden. Vielfach hat sich gezeigt, dass Krisen zu steigenden sozialen und geschlechtlichen Ungleichheiten führen können (z.B. OECD 2013).

Für die Legitimierung sozialpolitischer Reformen - und damit einhergehend: die Reduzierung oder Perpetuierung ökonomischer Ungleichheiten - spielen Narrative eine zentrale Rolle (Cox 2001). Sie transportieren eine bestimmte und i.d.R. vereinfachte Situationsbeschreibung und zeichnen sich durch bestimmte Strukturmerkmale aus (Stone 2012). Von zentraler Bedeutung ist hierbei die soziale Konstruktion von Zielgruppen sozialpolitischer Reformen (Schneider und Ingram 1993). Insbesondere bauen diese Konstruktionen auf einer Unterscheidung von ,verdienten“ (deserving) und ,unverdienten“ (undeserving) Gruppen auf, die dementsprechend mit höherer bzw. niedrigerer Wahrscheinlichkeit durch sozialpolitischen Aus- oder Rückbau adressiert werden (Blum und Kuhlmann 2019). Diese Zielgruppen-Konstruktionen sind ein zentrales Element im politischen Prozess und werden in Narrativen festgeschrieben, auch und gerade in Krisenzeiten (Boin et al. 2016; Kuhlmann und Blum 2021).

Dieser Beitrag untersucht, wie sozialpolitische Reformen in Zeiten akuter Krisen über Narrative kommuniziert werden, und wie darin zentrale Zielgruppen konstruiert werden. Dabei untersuchen wir zwei Policies, die sowohl in der Finanzkrise 2008/2009 als auch in der Corona-Krise prominent wurden: Zum einen die Kurzarbeit, die in der Corona-Pandemie, teilweise unter explizitem Rückgriff auf die Erfahrungen mit der Finanzkrise, als „Brücke“ über die Krise eingesetzt wurde (Deutscher Bundestag 2020d, S. 19712) und zum anderen das Elterngeld, das als „Deutschlands beliebteste Familienleistung“ (BMFSFJ 2020) in beiden Krisen angepasst wurde. Die entsprechenden Krisenreaktionen decken dabei überwiegend Expansions-, mit den Elterngeldreformen aber auch Retrenchment-Maßnahmen ab. Für letztere sind ein besonderer Legitimierungsbedarf und politische Kommunikationsstrategien zu erwarten (Pierson 1994; Wenzelburger und Hörisch 2016). Allerdings trafen die Finanzkrise und die Corona-Pandemie tendenziell unterschiedliche Gruppen. Anhand beider Policies soll daher untersucht werden, inwiefern sich die Narrative im Hinblick auf die Konstruktion der jeweiligen mit den Maßnahmen adressierten Gruppen - und damit auch die Legitimationsstrategien für unterschiedliche Ausmaße von Ungleichheit - unterschieden. Die Analyse basiert auf einer qualitativen Inhaltsanalyse von Dokumenten des Policy-Prozesses, von Pressemeldungen der zuständigen Ministerien sowie der Medienberichterstattung (SZ, FAZ).

Im folgenden Kapitel werden die für den sozialpolitischen Bereich beschriebenen unterschiedlichen Reformnarrative dargestellt. Kap. 3 gibt einen Überblick über das methodische Vorgehen. Mit Kap. 4 folgt die Beschreibung der Krisenreformen zur 
Kurzarbeit sowie die Analyse ihrer Krisennarrative (4.1), die analogen Ergebnisse für die Elterngeldreformen (4.2) sowie eine vergleichende Betrachtung (4.3). Kap. 5 zieht ein abschließendes Fazit.

\section{Sozialpolitische Reformnarrative}

Das Narrativ ist zu einer zunehmend wichtigen Kategorie in der Policy-Forschung avanciert. „We don't usually think of policy as literature, but most definitions of policy problems have a narrative structure" (Stone 2012, S. 158). Zentrales Element von Narrativen ist ihre Vereinfachung eines i.d.R. komplexen Sachverhalts (Münch 2016, S. 84-85); sie dienen dabei ganz wesentlich der ,Sinnvermittlung und Legitimitätserzeugung“ (Gadinger et al. 2014, S. 10). Dies geschieht typischerweise über kausale Verknüpfungen zwischen verschiedenen Ereignissen zu einer Erklärung darüber, wie ,die Welt funktioniert“. ${ }^{2}$ Stone (2012) identifiziert verschiedene Typen von Plots, die entweder Wandel (in Form von Niedergang oder Aufschwung) oder Macht (in Form von Kontrolle oder Nicht-Kontrolle bzw. Hilflosigkeit) hervorheben. ${ }^{3}$ Erzählungen des Niedergangs sind hierbei am häufigsten zu finden (Stone 2012, S. 160), da über die Konstruktion einer ,permanenten Verschlechterung“" politischer Handlungsdruck konstruiert werden kann, der nur durch die Einführung einer bestimmten Policy (als „Moral“ der Geschichte) aufgelöst oder sogar in einen „Aufschwung“ umgewandelt werden könne. Plots, die Macht in den Vordergrund stellen, fokussieren entweder darauf, wie Kontrolle über eine bestimmte Entwicklung wiedererlangt werden kann. Oder aber sie betonen umgekehrt die Unkontrollierbarkeit und dementsprechende eigene Hilflosigkeit (,Uns sind die Hände gebunden."). In Narrativen finden typische sprachliche Mittel Verwendung, wovon Stone (2012, S. 171) v. a. Symbole, Metaphern und Synekdochen herausarbeitet, bei denen eine Gesamtheit durch einen ,typischen Fall“ repräsentiert wird.

Angesichts hoher Popularität und großer Betroffenheit kommt Narrativen in der Sozialpolitik eine besondere Funktion zu, in denen spezifische inhaltliche Konstruktionen wirksam werden. Vor diesem Hintergrund haben wir in einem vorherigen Artikel (Blum und Kuhlmann 2019) eine Typologie sozialpolitischer Reformnarrative entwickelt, die es erlauben soll, die Unterschiede sozialpolitischer Narrative für unterschiedliche Reformtypen systematisch herauszuarbeiten, wobei neben Stones Arbeiten zu Narrativen auch die Arbeiten zur sozialen Konstruktion von Zielgruppen (Schneider und Ingram 1993; Ingram und Schneider 2015) eine Grundlage bilden. In diesem Artikel wenden wir die Typologie sozialpolitischer Reformnarrative erstmals in einer Fallstudie systematisch an.

\footnotetext{
2 Denn einer sozial-konstruktivistischen Epistemologie folgend werden reale Phänomene von Menschen ganz unterschiedlich gesehen und interpretiert, was sich in entsprechenden Narrativen spiegelt (vgl. Dodge 2015).

${ }^{3}$ Die Ausprägungen dieser Geschichten von Wandel bzw. Kontrolle schließen sich nicht gegenseitig aus, sondern können verknüpft werden.
} 


\subsection{Soziale Konstruktion von Gruppen nach Schneider und Ingram}

Das Kernargument von Schneider und Ingram lautet, dass Gruppen im politischen Prozess nicht ausschließlich im Hinblick darauf relevant sind, ob es sich bei ihnen um mächtige oder weniger mächtige Gruppen handelt, sondern dass ebenfalls ausschlaggebend ist, ob die jeweilige Gruppe als positiv oder negativ, als „verdient“ oder ,unverdient“ angesehen wird. Entsprechend kommt der sozialen Konstruktion von Gruppen eine besondere Bedeutung zu. Schneider und Ingram (1993, S. 334) beschreiben dies als ,cultural characterizations or popular images of the persons or groups whose behavior and well-being are affected by public policy. These characterizations are normative and evaluative, portraying groups in positive or negative terms through symbolic language, metaphors, and stories." Die Konstruktion von Gruppen ist somit ein heftig umkämpfter und in gewisser Weise immer auch kontingenter politischer Prozess.

Auf Grundlage dieser Überlegungen unterscheiden Schneider und Ingram vier Gruppen:

- Advantaged („,Begünstigte“): mächtig und positiv konstruiert (z. B. Ältere, Mittelschicht)

- Contenders („Privilegierte“): mächtig aber negativ konstruiert (z. B. Reiche)

- Dependents (,Abhängige“): schwach aber positiv konstruiert (z.B. Mütter, Kinder, arme Familien)

- Deviants (,Abweichler“): schwach und negativ konstruiert (z. B. Drogenabhängige, illegale Migrantinnen)

In sozialpolitischen Reformnarrativen werden nun Gruppen mit bestimmten Attributen versehen und somit begründet, ob Leistungen, die dieser Gruppe gewährt oder verwehrt werden, als verdient oder unverdient angesehen werden. Verschiedene Arten von Reformen betreffen dabei unterschiedliche Zielgruppen, die jedoch nicht einfach in klaren Konturen existieren, sondern unter anderem durch Narrative konstruiert werden können. Die Bedeutung bestimmter Charaktere und Gruppendarstellungen für politische Narrative ist von verschiedenen Autorinnen hervorgehoben worden. So hat beispielsweise Husmann (2015, S. 420) argumentiert, dass Advantaged in Narrativen typischerweise als „Heldinnen“ auftreten, während Dependents die Rolle unschuldiger „Opfer“ zukommt und Contenders sowie Deviants als „Schurkinnen“ portraitiert werden. Diese Erwartungen sind angesichts der Zielgruppencharakteristika plausibel, aber auch alternative Darstellungen denkbar, z. B. durch Arbeitsunfälle bedrohte Advantaged als Opfer oder Mütter als heldinnenhaft dargestellte Dependents.

\subsection{Typologie sozialpolitischer Reformnarrative}

In der Typologie gehen wir davon aus, dass Narrative systematische Unterschiede aufweisen abhängig davon, 1) ob sie sich auf Aus- oder Rückbau sozialpolitischer 
Tab. 1 Typen sozialpolitischer Reformnarrative (Quelle: Blum und Kuhlmann 2019; auf Grundlage von Stone 2012, Schneider und Ingram 1993)

\begin{tabular}{|c|c|c|}
\hline \multirow[t]{2}{*}{ Kontext } & \multicolumn{2}{|l|}{ Reformen } \\
\hline & Expansion & Retrenchment \\
\hline $\begin{array}{l}\text { Alte so- } \\
\text { ziale }\end{array}$ & $\begin{array}{l}\text { (I) Erzählungen vom giving- } \\
\text { to-give }\end{array}$ & $\begin{array}{l}\text { (II) Erzählungen vom taking-to-take oder taking-to- } \\
\text { control }\end{array}$ \\
\hline Risiken & $\begin{array}{l}\text { Verdientheit und Anerken- } \\
\text { nung (Advantaged) }\end{array}$ & $\begin{array}{l}\text { Unverdientheit und Eigenverantwortung (Deviants; } \\
\text { Contenders) } \\
\text { Hilflosigkeit und Kontrolle (Advantaged) }\end{array}$ \\
\hline $\begin{array}{l}\text { Neue } \\
\text { soziale }\end{array}$ & $\begin{array}{l}\text { (III) Erzählungen vom } \\
\text { giving-to-promote }\end{array}$ & $\begin{array}{l}\text { (IV) Erzählungen vom taking-to-take oder taking- } \\
\text { out-of-helplessness }\end{array}$ \\
\hline Risiken & $\begin{array}{l}\text { Verdientheit und Befähigung } \\
\text { (Dependents) }\end{array}$ & $\begin{array}{l}\text { Unverdientheit (Deviants; Contenders) } \\
\text { Hilflosigkeit (Dependents) }\end{array}$ \\
\hline
\end{tabular}

Während wir in Blum und Kuhlmann (2019) von giving-to-shape-Narrativen gesprochen haben, sprechen wir nun von giving-to-promote-Narrativen (vgl. auch Kuhlmann und Blum 2021), da dieser Begriff den zentralen Aspekt der Befähigung (s.u.) besser abbildet

Leistungen beziehen, ${ }^{4}$ und 2) ob sie Policies gegen sogenannte alte oder neue soziale Risiken (s.u.) betreffen. Aus diesen theoretischen Überlegungen ergibt sich eine Vier-Felder-Matrix (Tab. 1) mit verschiedenen Reformnarrativen, den darin zu erwartenden Charakteren sowie den ihnen zugeschriebenen Attributen. Bei diesen unterschiedlichen Narrativen nach Reformtyp handelt es sich um Idealtypen, von denen in realen Kontexten immer auch Abweichungen erwartbar sind (zum Folgenden vgl. ausführlicher Blum und Kuhlmann 2019).

Idealtypisch stehen zunächst Reformen klassischer Policies gegen alte soziale Risiken (old social risks: OSR) im Fokus, die entweder in Richtung Expansion oder Rückbau gehen können. OSR entstehen durch die traditionellen „Wechselfälle des Lebens", wie Alter, Krankheit oder Arbeitslosigkeit, die typischerweise über monetäre Kompensation adressiert werden (Bonoli 2005). Von etablierten sozialen Sicherungssystemen zur OSR-Absicherung profitieren v. a. traditionelle (i.d.R. männliche) Normalarbeitnehmer, die ,labour market insiders“ (Schwander und Häusermann 2013), die demnach Charakteristika der Advantaged aufweisen.

(I) Wenn OSR-Policies gestärkt werden, ist folglich zu erwarten, dass diese vor allem für die Gruppe der Advantaged gelten, die positiv konstruiert und zugleich mächtig sind, d.h. auch relevante (Wähler-)Gruppen darstellen. In giving-to-giveNarrativen zur Reformlegitimation steht dabei vor allem die Verdientheit dieser Gruppen im Vordergrund, insbesondere vor dem Hintergrund der von ihnen erbrachten Leistungen (bei Rentnerinnen beispielsweise: „Menschen, die ihr Leben lang hart gearbeitet haben."). Ein konkretes Beispiel für solche Narrative ist empirisch in den Debatten um die Rente mit 63 in Deutschland (2014) zu finden, in denen ein früherer Renteneintritt als Anerkennung für die von diesen Gruppen geleistete Arbeit konstruiert wurde (Blum 2019).

\footnotetext{
${ }^{4}$ Hier ist wichtig zu ergänzen, dass dies für einzelne Reformelemente unterschiedlich ausfallen kann. Für Details zur Begründung der vier verschiedenen Reformtypen siehe Blum und Kuhlmann (2019).
} 
(II) Für den Rückbau von OSR-Policies hingegen gewinnen Narrative an Bedeutung, die Zielgruppen als unverdient konstruieren, v. a. im Feld der Arbeitsmarktpolitik und sozialen Sicherung, um damit eine Kürzung sozialpolitischer Leistungen zu legitimieren. Dies findet statt über taking-to-take-Narrative. Eng verbunden ist hiermit das Schlagwort der Eigenverantwortung. Ist eine narrative Konstruktion als „unverdiente“ Deviants oder Contenders hingegen nicht möglich bzw. politisch zu riskant, so stehen Konstruktionen zu erwarten, die auf externe und widrige Umstände rekurrieren (z. B. „Sparzwänge“), aufgrund derer nur durch entschlossenes Handeln (aller) die Kontrolle zurückgewonnen werden kann, was sich sprachlich in takingto-control-Narrativen niederschlägt.

Demgegenüber stehen Policy-Reformen im Bereich neuer sozialer Risiken (new social risks: NSR), die sich auf Problemlagen der sog. post-industriellen Gesellschaften beziehen (z. B. Vereinbarkeit von Familie und Beruf, Niedrigqualifizierung) und die ebenfalls in Richtung Expansion oder Rückbau gehen können. Bonoli (2005) hat argumentiert, dass Gruppen, die NSR ausgesetzt sind, politisch unterrepräsentiert sind. Er führt Frauen, junge Menschen sowie Niedrigqualifizierte als relevante Gruppen an. Mit Schneider und Ingram (1993) können diese Gruppen als Dependents - oder aber auch als Deviants - konstruiert werden.

(III) Narrative, die den Ausbau von NSR-Policies legitimieren sollen, heben vor allem die Verdientheit der betroffenen Gruppen hervor. Da die Bekämpfung von NSR zudem im Kontext der Debatten um einen ,neuen Wohlfahrtsstaat“ (Esping-Andersen et al. 2002) sowie einer Social-investment-Strategie steht (Hemerijck 2015), können giving-to-promote-Narrative erwartet werden, die vor allem das Potenzial zur Befähigung hervorheben.

(IV) Beim Rückbau von NSR-Policies ist hingegen von erschwerten Legitimationsbedingungen auszugehen. Wenn die Gruppe der als Dependents Konstruierten von Abbau betroffen ist, können taking-out-ouf-helplessness-Narrative dominieren. Alternativ können die von Rückbau betroffenen Gruppen in taking-to-take-Narrativen auch als unverdient charakterisiert werden. So können Langzeitarbeitslose in Narrativen als Deviants konstruiert werden, die sich in der ,sozialen Hängematte“ ausruhten.

\section{Methodisches Vorgehen}

Für die Analyse der Narrative, die zur Legitimierung der Reformen verwendet wurden, ziehen wir unterschiedliche Materialien heran. Zum einen wurden Dokumente des Policy-Prozesses (z. B. Plenarprotokolle des Deutschen Bundestages) sowie Pressemeldungen der jeweiligen Ministerien einbezogen. Da politische Akteure zur Reformkommunikation auch (traditionelle) Massenmedien nutzen bzw. Medien über die Reformen und deren stattfindende Legitimation berichten, wurde außerdem die Berichterstattung in der Süddeutschen Zeitung (SZ) sowie der Frankfurter Allgemeine Zeitung (FAZ) erhoben, wobei der SZ tendenziell eine liberalere und der FAZ tendenziell eine konservativere Ausrichtung zugeschrieben wird. Das Material wur- 
Tab. 2 Anzahl ausgewerteter Zeitungsartikel und Plenarprotokolle

\begin{tabular}{|c|c|c|c|}
\hline & Zeitraum & Zeitungsartikel & Plenarprotokolle \\
\hline $\begin{array}{l}\text { Elterngeld } \\
2010\end{array}$ & $01.06 .2010-31.12 .2010$ & $\begin{array}{l}\text { Treffer insgesamt: } 189 \text { (116 SZ; } 73 \text { FAZ) } \\
\text { Einbezogen: } 37 \text { (18 SZ; } 19 \text { FAZ) }\end{array}$ & Einbezogen: 7 \\
\hline $\begin{array}{l}\text { Elterngeld } \\
2020 / 2021\end{array}$ & 01.02.2020-10.2.2021 & $\begin{array}{l}\text { Treffer insgesamt: } 46 \text { (19 SZ; } 27 \text { FAZ) } \\
\text { Einbezogen: } 3 \text { ( } 2 \text { SZ; } 1 \text { FAZ) }\end{array}$ & Einbezogen: 5 \\
\hline $\begin{array}{l}\text { Kurzarbeit } \\
2008 / 2009\end{array}$ & 15.10.2008-31.1.2009 & $\begin{array}{l}\text { Treffer insgesamt: } 578 \text { ( } 369 \text { SZ; } 209 \text { FAZ) } \\
\text { Einbezogen: } 14 \text { (8 SZ; } 6 \text { FAZ) }\end{array}$ & Einbezogen: 11 \\
\hline $\begin{array}{l}\text { Kurzarbeit } \\
2020\end{array}$ & $01.03 .2020-31.5 .2020$ & $\begin{array}{l}\text { Treffer insgesamt: } 1122 \text { (706 SZ; } 416 \text { FAZ) } \\
\text { Einbezogen: } 14 \text { (6 SZ; } 8 \text { FAZ) }\end{array}$ & Einbezogen: 7 \\
\hline
\end{tabular}

de über eine breite Schlüsselwortsuche (,Elterngeld“; „,Kurzarbeit“) eingegrenzt. Für das Medienmaterial wurde die Berichterstattung für die jeweiligen Zeiträume vom Agenda-Setting bis zur Beschlussfassung recherchiert. Daraufhin wurden die Artikel auf ihre Relevanz hin gesichtet und in den Artikelkorpus einbezogen, wenn in ihnen zumindest ein direktes oder indirektes Zitat eines politischen Akteurs der Bundesebene zu der jeweiligen Reform enthalten war. Für die Plenarprotokolle wurden für das Elterngeld relevante Bundestagsdebatten zu seinen Reformen ausgewertet. Für die Kurzarbeit wurden aufgrund der größeren Rolle des Instruments im gesamten Krisenkontext breiter Plenarprotokolle im Untersuchungszeitraum einbezogen, um auch die Legitimation von Kurzarbeit im Zusammenhang mit anderen verabschiedeten Maßnahmen zu erfassen. ${ }^{5}$

Tab. 2 gibt eine Übersicht über die Schritte zur Materialeingrenzung und die analysierten Dokumente.

Das Material wurde mithilfe von MAXQDA einer qualitativen Inhaltsanalyse unterzogen. Aus den theoretischen Vorüberlegungen wurden hierbei Kategorien zu den Zielgruppen sowie Expansions- und Rückbaunarrativen gebildet. Zudem wurden weitere Kategorien zu den konkret verwendeten Argumentationsmustern der politischen Akteure gebildet, die induktiv während der Extraktion hinzugefügt wurden.

\section{Reformen und narrative Strategien in Krisenzeiten}

\subsection{Das Kurzarbeitergeld}

\subsubsection{Krisenreformen der Kurzarbeit}

Beim Kurzarbeitergeld handelt es sich um eine Einkommensersatzleistung zur Kompensation von Einkommenseinbußen infolge von Kurzarbeit (Bäcker et al. 2020, S. 487), die von der Bundesagentur für Arbeit gezahlt wird. Betriebe können für ihre sozialversicherungspflichtig Beschäftigten Kurzarbeit anmelden, wenn ein ,erheblicher Arbeitsausfall“ (SGB III §95) vorliegt, der auf „wirtschaftlichen Gründen“ oder einem ,,unabwendbaren Ereignis“ beruht, ,,vorübergehend“ und ,nicht vermeid-

\footnotetext{
5 Beim Instrument der Kurzarbeit wurde aufgrund der großen Anzahl der potenziell relevanten Debatten und Zeitungsartikel nur der Zeitraum bis Ende Mai 2020, d. h. die sog. erste Pandemiewelle, ausgewertet.
} 
bar" ist (SGB III §96). Zudem muss in einem Betrieb bei mindestens einem Drittel der Beschäftigten ein Entgeltausfall von mindestens $10 \%$ des monatlichen Bruttoentgelts vorliegen (SGB III § 96).

Kurzarbeit dient zwei Zielen: Als Einkommensersatzleistung erfüllt das Kurzarbeitergeld für Beschäftigte eine sozialpolitische Funktion. Die Leistung wird i.d.R. für bis zu zwölf Monate für die ausgefallenen Arbeitsstunden gezahlt. Die Höhe des Kurzarbeitergeldes beträgt - analog zum Arbeitslosengeld I - 60\% des ausgefallenen pauschalierten Nettoeinkommens; für Beschäftigte mit Kind(ern) erhöht sich der Betrag auf $67 \%$ (Bäcker et al. 2020, S. 488). Darüber hinaus erfüllt die Kurzarbeit aber auch eine wirtschaftspolitische Funktion, indem sie Betrieben ermöglicht, ihre Beschäftigten in Krisenzeiten zu halten. Vor allem in koordinierten Marktwirtschaften ist die Kurzarbeit somit ein wichtiges Instrument zum Erhalt von „Humankapital“ (Sacchi et al. 2011, S. 466). Mit dem Kurzarbeitergeld wird das OSR Arbeitslosigkeit adressiert. In Bezug auf die Zielgruppen sozialpolitischer Maßnahmen adressiert die Kurzarbeit primär Advantaged, d.h. gut ausgebildete, sozialversicherungspflichtig beschäftigte Arbeitnehmerinnen, an deren Verbleib im Betrieb Arbeitgeberinnen interessiert sind.

Kurzarbeit hatte in den letzten Jahrzehnten regelmäßig eine große arbeitsmarktpolitische Bedeutung inne. Nach der Wirtschafts- und Finanzkrise, in der 2009 über 1,1 Mio. Beschäftigte Kurzarbeitergeld bezogen, wurde es als maßgeblich für die relativ milden Krisenauswirkungen auf den deutschen Arbeitsmarkt angesehen (Schulten und Müller 2020, S. 4-5). Im Zuge der beiden Konjunkturpakete wurden einige zeitlich befristete Erleichterungen bei der Kurzarbeit beschlossen: So wurde der Zugang deutlich erleichtert, indem auch Betriebe Kurzarbeit beantragen konnten, in denen ein oder mehrere Mitarbeiter einen Entgeltausfall von mindestens $10 \%$ haben. Zudem wurde die Bezugsdauer von Kurzarbeitergeld zunächst auf 18 Monate (November 2008) und dann auf 24 Monate (Januar 2009) ausgedehnt. Die Bundesagentur für Arbeit erstattete außerdem den Betrieben 50\% der Sozialversicherungsbeiträge, und $100 \%$ bei Qualifizierungsmaßnahmen für Beschäftigte. Zudem wurden Leiharbeiter in die Kurzarbeiterregelungen einbezogen (Sacchi et al. 2011; Dümig 2010) und dadurch das wesentlich für „Arbeitsmarktinsider“ (Advantaged) eingesetzte Instrument auf eine weitere Gruppe ausgedehnt, die aufgrund ihrer schwachen Machtressourcen (Fervers und Schwander 2015) als Dependents charakterisiert werden kann.

$\mathrm{Zu}$ Beginn der Corona-Pandemie wurden erneut die Zugangsbedingungen zur Kurzarbeit erleichtert, wobei größtenteils ähnliche Regelungen wie während der Finanzkrise getroffen wurden (vgl. zum Folgenden BMAS 2021, Stand: Dezember 2021). Es können auch Betriebe Kurzarbeit beantragen, in denen mindestens $10 \%$ der Beschäftigten einen Einkommensausfall erleiden. Die Bezugsdauer des Kurzarbeitergeldes wurde auf bis zu 24 Monate und maximal bis zum 31. März 2022 verlängert. Betrieben werden Sozialversicherungsbeiträge bis Ende 2021 vollständig erstattet. ${ }^{6}$ Durch eine Änderung des Arbeitnehmerüberlassungsgesetzes wurde Kurz-

\footnotetext{
6 Die Erstattung der Sozialversicherungsbeiträge beträgt ab Januar 2022 bis März 2022 nur noch $50 \%$. Eine partielle Erstattung der Beiträge bis Ende Juli 2023 ist möglich, wenn Beschäftigten ermöglicht wird, sich während Kurzarbeit weiterzubilden. Auch können Betrieben Kosten für Lehrgänge erstattet werden.
} 
arbeit zudem auch für Leiharbeitnehmerinnen möglich. Neue Regelungen wurden im Zuge der Sozialschutzpakete getroffen: Das erste Sozialschutzpaket sah erleichterte Zuverdienstmöglichkeiten für Beschäftigte in Kurzarbeit vor. Mit dem zweiten Sozialschutzpaket wurde die Höhe des Kurzarbeitergeldes angehoben, wenn Beschäftigte einen Entgeltausfall von zumindest $50 \%$ haben. Ab dem vierten Monat erhöht sich das Kurzarbeitergeld auf $70 \%$ (77\% mit Kindern), ab dem siebten Monat auf $80 \%$ (87\% mit Kindern). Die Regelungen zur Höhe des Kurzarbeitergeldes wurden mit wenigen Anpassungen bis zum 31. März 2022 verlängert. Im Mai 2020 bezogen 7,3 Mio. Beschäftigte Kurzarbeitergeld. ${ }^{7}$

Bezugszahlen zeigen, dass Kurzarbeitergeld in beiden Krisen von sehr unterschiedlichen Berufsgruppen in Anspruch genommen wurde: In der Finanzkrise bezogen v. a. Beschäftigte im verarbeitenden Gewerbe und der Exportindustrie - beispielsweise der Autobranche - Kurzarbeitergeld, d.h. in traditionell gut bezahlten Beschäftigungsverhältnissen. In der Corona-Pandemie hingegen wird das Instrument der Kurzarbeit in vielen Branchen genutzt; besonders häufig in der Gastronomie und im Dienstleistungssektor, in denen Arbeitnehmerinnen i.d.R. weniger verdienen. Auffällig ist zudem, dass im Bereich der ,,sonstigen wirtschaftlichen Dienstleistungen“ (inkl. Leiharbeit) der Bezug von Kurzarbeitergeld deutlich höher ist als während der Finanzkrise (Gehrke und Weber 2020).

\subsubsection{Krisennarrative zum Kurzarbeitergeld}

Finanzkrise In der Finanzkrise nutzten politische Regierungsakteure vor allem giving-to-promote-Narrative, um die Erleichterungen beim Kurzarbeitergeld argumentativ zu untermauern. Das bedeutet, dass Reformen beim Kurzarbeitergeld als Mittel zur Befähigung legitimiert wurden. Diese Befähigung bezog sich zum einen auf die Betriebe, an die eindringlich appelliert wurde, Kurzarbeit anzumelden (SZ 2009) und deren Kapazitäten durch die Kurzarbeit erhalten werden sollten, und zum anderen auf die Arbeitnehmerinnen - überwiegend sog. Arbeitsmarktinsider -, die Kurzarbeit beziehen und denen im Rahmen von Kurzarbeit die Möglichkeit zur Weiterqualifizierung gegeben werden sollte. Beide Gruppen können mit Schneider und Ingram (1993) den Advantaged zugerechnet werden.

Sprachlich wurde das Instrument der Kurzarbeit als „Brücke“ (Merkel, Deutscher Bundestag 2008c, S. 20337) hinüber in die Zeit nach der Krise charakterisiert. Es gebe, so Nahles einen Tag vor ihrer Wahl zur SPD-Generalsekretärin, „Unternehmen, die Sorge haben [...]. Wenn diese Unternehmen ihre Leute jetzt entlassen müssen, finden sie später keine guten Leute. Damit wäre der gesamte Betrieb gefährdet. [...] Deshalb ist es so wichtig, die Bezugsdauer des Kurzarbeitergeldes zu verlängern.“ (Deutscher Bundestag 2008b, S. 19874). In diesem Zitat wird der Aspekt der Befähigung besonders deutlich, indem nicht auf bisherige Leistungen oder eine allgemeine Anerkennung der grundsätzlich wichtigen Rolle der Betriebe für das deutsche Wirtschaftssystem Bezug genommen wird, sondern auf die Notwendigkeit des Erhalts ihrer Betriebsstärke und die Vermeidung von Entlassungen, um nach der Krise mit der gleichen Belegschaft weiterarbeiten zu können.

\footnotetext{
7 https://de.statista.com/statistik/daten/studie/2603/umfrage/entwicklung-des-bestands-an-kurzarbeitern/.
} 
Vor allem sollten Betriebe die Zeit der Kurzarbeit für die Weiterqualifizierung ihrer Mitarbeiterinnen nutzen, wodurch durch die vollständige Erstattung der Sozialversicherungsbeiträge Anreize gesetzt wurden. Der SPD-Abgeordnete Stiegler bezeichnete die Dauer der Kurzarbeit besonders bildhaft als ,Trainingslager [...] für den nächsten Aufschwung“ (Deutscher Bundestag 2008c, S. 20363). „Qualifizieren statt entlassen“ (Deutscher Bundestag 2008a, S. 19784) wurde zu einem wesentlichen Topos innerhalb des giving-to-promote-Narrativs, der sowohl von Finanzminister Steinbrück (SPD) als auch vom damaligen Arbeitsminister Scholz (SPD), teilweise verbunden mit direkten Appellen an die Unternehmen, genutzt wurde und den Aspekt der Befähigung der Arbeitnehmerinnen - vor allem vor dem Hintergrund der Befähigung der Betriebe - in den Vordergrund stellte:

Halten Sie die Arbeitsplätze! Diese gut qualifizierten oder zu qualifizierenden Arbeitnehmerinnen und Arbeitnehmer brauchen Sie aufgrund der weiteren demografischen Entwicklung in zwei, drei Jahren dringend. Deshalb setzen Sie sie nicht auf die Straße, sondern nehmen Sie die Qualifizierungsangebote, die es gibt, an (Steinbrück; Deutscher Bundestag 2008a, S. 19784).

Neben die giving-to-promote-Narrative treten allerdings auch giving-to-give-Narrative, wenngleich weniger prominent, die Arbeitnehmerinnen in Kurzarbeit als Gruppe mit legitimem finanziellen Unterstützungsbedarf konstruieren. Arbeitnehmerinnen sollen durch Kurzarbeitergeld vor Arbeitslosigkeit bewahrt und finanziell abgesichert werden: „Denjenigen, die jetzt arbeitslos würden, zum Beispiel in der Stahlbranche, können wir Kurzarbeitergeld anbieten. Das ist zwar weniger, als sie bisher verdient haben [...]; aber es nützt den Menschen mehr, wenn sie ihren Job behalten, als wenn sie arbeitslos werden, einen höheren Hartz-IV-Satz erhalten und zu Hause sitzen." (Nahles, Deutscher Bundestag 2009a, S. 21449) Dieses giving-togive-Narrativ wird teilweise auch mit einem giving-to-promote-Narrativ verknüpft, indem auf Weiterqualifizierung während der Kurzarbeit hingewiesen wird. Nahles' Verweis auf die starke Stahlbranche deutet bereits auf Advantaged als zentrale Zielgruppe in Narrativen zur Kurzarbeit hin. Der CDU-Abgeordnete Nüßlein charakterisiert die Kurzarbeit sogar explizit als Instrument zur Unterstützung des Mittelstandes: „Ich möchte ausdrücklich darauf hinweisen, dass es nicht nur die Großen sind, die geschützt werden, sondern dass wir das Augenmerk auch auf den Mittelstand richten." (Deutscher Bundestag 2009b, S. 21984) Dies ist auch vor dem Hintergrund der v.a. von der Linkspartei geäußerten Kritik zu verstehen, dass in der Finanzkrise primär große Unternehmen und Banken gerettet würden.

Insbesondere im Zusammenhang mit giving-to-give-Narrativen werden aber auch die Leiharbeiterinnen als Dependents adressiert, die während der Finanzkrise vor Entlassungen geschützt werden sollen.:

Wir merken doch auch, wer jetzt wieder der Erste ist, der dran glauben muss. Das sind die, die ohnehin schon prekär beschäftigt sind, nämlich die Leiharbeiter. [...] Auch diejenigen, die wir immer wieder ein Stück weit unter Druck setzen, müssen von uns die Hand gereicht bekommen. Auch sie müssen von uns ein Signal bekommen, dass uns ihr Schicksal nicht egal ist. Deswegen bin 
ich sehr froh, dass wir es angehen, auch Leiharbeitern den Bezug von Kurzarbeitergeld zu ermöglichen (Nahles, Deutscher Bundestag 2008d, S. 20481).

Coronakrise $\mathrm{Zu}$ Pandemie-Beginn kündigte Scholz - nun Finanzminister - an, wenn es um die Abmilderung der wirtschaftlichen Folgen der Corona-Pandemie gehe, solle „keine einzige Hilfsmaßnahme am Geld scheitern“ (SZ 2020a). Auch Arbeitsminister Heil (SPD) ließ verlauten: ,Wir werden um jeden Arbeitsplatz kämpfen." (Deutscher Bundestag 2020a, S. 19160) In diesem Zusammenhang kommt dem Kurzarbeitergeld als erste in der Corona-Pandemie verabschiedete sozialpolitische Maßnahme eine prominente Rolle zu. Kanzlerin Merkel hob es als erfolgreichen Bestandteil der Bekämpfung der wirtschaftlichen und sozialen Folgen der CoronaPandemie positiv hervor: „Dass Menschen in Kurzarbeit sind [...], zeigt, dass wir die Menschen nicht ins Nichts entlassen mussten, sondern dass wir helfen können“ (Deutscher Bundestag 2020d, S. 19696).

Ähnlich wie in der Finanzkrise wird das Kurzarbeitergeld auch jetzt über givingto-give- und giving-to-promote-Narrative legitimiert (vgl. auch Blum und Kuhlmann 2021). Allenfalls angedeutet werden zukünftige Kürzungen (im Sinne eines takingto-control-Narrativs) an einer Stelle vom CDU-Fraktionsvorsitzenden Brinkhaus: „wir müssen eine Sache vielleicht auch mal beachten: All das, was wir beschließen [...], kostet Geld - viel Geld -, das von irgendjemandem mal wieder zurückgezahlt werden muss." (Deutscher Bundestag 2020c, S. 19307). Deutlich erkennbar werden in den giving-to-give- und giving-to-promote-Narrativen Rückbezüge auf den deutschen Sozialstaat bzw. die soziale Marktwirtschaft als Heldin, auf die gerade in Krisenzeiten Verlass sei. Ein Zitat von Wirtschaftsminister Altmeier (CDU) verdeutlicht, wie in der Logik der sozialen Marktwirtschaft der Erhalt von Betrieben (legitimiert über giving-to-promote-Narrative, da der Erhalt der Wirtschaftskraft der Betriebe im Vordergrund steht) und die soziale Absicherung von Arbeitnehmerinnen (legitimiert über giving-to-give-Narrative, da die notwendige finanzielle Unterstützung dieser verdienten Gruppe im Vordergrund steht) zwei Seiten einer Medaille sind, die mit dem Instrument der Kurzarbeit erreicht werden können:

Die soziale Marktwirtschaft ist deshalb so genial, weil sie die freie Entfaltung des Unternehmertums ganz obenan stellt, aber die Schwachen und diejenigen, die Hilfe brauchen, niemals alleine und im Stich lässt. Deshalb [...] bin ich sehr dankbar, dass wir [...] über Parteigrenzen hinweg erkannt haben, dass die Verlängerung und die Verbesserung von Kurzarbeit eben eine Maßnahme ist, die nicht nur der Wirtschaft oder den Arbeitnehmern nutzt, sondern allen gemeinsam. Sie erhält Arbeitsplätze; sie erhält Betriebe; sie ermöglicht es, die Durststrecken zu überwinden (Deutscher Bundestag 2020a, S. 19135).

Giving-to-give-Narrative finden sich v. a. im Zusammenhang mit der im Sozialschutz-Paket II geregelten Aufstockung des Kurzarbeitergeldes: „diese Beschäftigten [müssen], wenn sie sehr lange in Kurzarbeit sind, erhebliche Gehaltseinbußen hinnehmen [...], während laufende Kosten - Mieten, Pachten, Zinsen, Kredite weiterlaufen. Deshalb ist es ein wichtiges Signal, dass diese Koalition mit den veränderten Regeln zur Kurzarbeit auch unverhältnismäßige Lohneinbußen abfedern 
wird.“ (Heil, Deutscher Bundestag 2020c, S. 19370). Während hier Arbeitnehmer als gesamte Gruppe als Advantaged charakterisiert werden können, zeigt die folgende Äußerung vom stellvertretenden Sprecher der Arbeitsgruppe Arbeit und Soziales der SPD-Fraktion, Rosemann, dass gerade Arbeitnehmerinnen, die wenig verdienen und daher auch als Dependents angesehen werden können, hier adressiert werden sollen: Die Aufstockung des Kurzarbeitergeldes sei „,vor allem für die Beschäftigten wichtig, die nur geringe Einkommen haben“ (Deutscher Bundestag 2020c, S. 19377). Auch erleichterte Zuverdienstmöglichkeiten bei Kurzarbeitergeld werden mithilfe von giving-to-give-Narrativen legitimiert: „Wir [vergrößern] die Zuverdienstmöglichkeiten für Menschen, die in Kurzarbeit sind, [...] damit Lohnlücken abgefedert werden.“ (Heil, Deutscher Bundestag 2020a, S. 19161). Als Beispiel führt Heil Wäscherei-Beschäftigte an, die bei Lohnausfällen aufgrund geschlossener Hotels in Krankenhäusern in der Wäscherei arbeiten können.

Die Argumentation, dass Beschäftigte den Kurzarbeitergeldbezug für Weiterbildung nutzen sollten, wurde erst im Rahmen des im Dezember 2020 verabschiedeten Beschäftigungssicherungsgesetzes prominenter. Betrieben würden über die hälftige Erstattung von Sozialversicherungsbeiträgen Anreize gesetzt, ,wo immer es möglich ist, Kurzarbeit mit Qualifizierung zu verbinden“ und „,dafür zu sorgen, dass die Beschäftigten von heute die Chance haben, die Arbeit von morgen zu machen“ (Heil, Deutscher Bundestag, 2020f, S. 24383). Dieses giving-to-promote-Narrativ verknüpft Heil auch mit einem Seitenhieb auf die Unternehmen als mögliche „Schurken“: „Was ich nicht erleben will, ist, dass nach der Krise, nach Massenentlassungen, dann über Fachkräftemangel gejammert wird.“ (ebd.)

Nicht zu übersehen sind schließlich explizite Bezüge auf die Kurzarbeit als bewährtes Instrument der Finanzkrise, teilweise unter Verwendung des gleichen Symbols der „Brücke über schwere Zeiten“ (z. B. BMAS-Staatssekretärin Griese, Deutscher Bundestag 2020d, S. 19712):

Dass Deutschland durch die letzte Krise 2008/2009 so gut gekommen ist, hat ganz wesentlich an dem automatischen Stabilisator Kurzarbeit gelegen, der dazu geführt hat, dass nicht plötzlich Millionen Arbeitnehmerinnen und Arbeitnehmer ihren Arbeitsplatz verloren haben. International wird es gelobt: vom Internationalen Währungsfonds, von der OECD, auch von der Europäischen Union schon immer in der Vergangenheit. Deshalb finde ich, es ist eine gute Botschaft, zu sagen: Alle wollen das jetzt auch machen (Scholz, Deutscher Bundestag 2020e, S. 20467f.).

Im Zusammenhang mit europäischen Initiativen zur Abfederung der Krise wird die Kurzarbeit hier als „Erfahrung der Sozialpartnerschaft aus Deutschland“ (ebd.) herausgestellt, die in anderen Ländern nun Nachahmerinnen finde.

\subsection{Das Elterngeld}

\subsubsection{Krisenreformen des Elterngelds}

Beim Elterngeld handelt es sich um eine steuerfinanzierte Geldleistung, die nach der Geburt eines Kindes für i. d. R. maximal 14 Monate gezahlt wird, wobei ein Elternteil 
(mit Ausnahme von Alleinerziehenden) maximal zwölf Monate in Anspruch nehmen kann. Zwar ist die Einkommensersatzlogik ${ }^{8}$ eine Kernidee des Elterngelds im Unterschied zur Pauschalleistung des vorherigen Erziehungsgelds. Allerdings wurde dessen vorherige Logik nicht komplett abgeschafft, sondern Nicht-Erwerbstätige erhalten weiterhin ein Mindestelterngeld von $300 € . .^{9}$ Dieser Hybridcharakter ist hier relevant, da das Elterngeld dementsprechend unterschiedliche Zielgruppen adressiert. Auf der einen Seite zielt das einkommensabhängige Elterngeld darauf ab, eine etwa einjährige „Familienphase“ nach der Geburt zu ermöglichen und danach eine Rückkehr von insbesondere Müttern (als Dependents) in den Beruf zu fördern, diese also zu ,,befähigen“. Es entspricht somit einem sozialinvestiven Ansatz (Nygard und Krüger 2012). Auf der anderen Seite stellt das Mindestelterngeld quasi ein „Überbleibsel" des vorherigen Erziehungsgelds, einer relativen niedrigen Pauschalleistung, die (wiederum insbesondere für Mütter) eine vergleichsweise lange Familienphase ermöglichen sollte. Diese Unterstützung weiblicher Sorgearbeit ist auch als „(p)art and parcel of the Keynesian-Beveridgean welfare compromise“ (Hemerijck 2015) beschrieben worden. Sie adressiert daher (traditionell) eher Advantaged, nämlich als Gegenstück des „männlichen Normalarbeitnehmers“ in Familienkonstellationen, die sich eine entsprechende Aufteilung von Erwerbs- und Sorgearbeit leisten können.

In der Finanz- und Wirtschaftskrise kam die Elterngeldreform im Rahmen des „Sparpakets“ der schwarz-gelben Bundesregierung auf die Agenda. Sie wurde mit Art. 14 des Haushaltsbegleitgesetzes am 09.12.2010 beschlossen und enthielt drei Kürzungsmaßnahmen. Diese Kürzungsmaßnahmen tangierten unterschiedliche Zielgruppen.

1. Zunächst wird das Elterngeld seit 01.01.2011 (ebenso wie zuvor z. B. schon Kindergeld) voll auf die Grundsicherungsleistungen von Hartz-IV-Empfängerinnen angerechnet, de facto wurde ihnen die zusätzliche Leistung hiermit gestrichen. Diese Gruppe der Langzeitarbeitslosen wird nach Schneider und Ingram (1993; vgl. auch Green-Pedersen 2002) als Dependents oder aber als Deviants konstruiert.

2. Zweitens wurde die Einkommensersatzrate für Nettoeinkommen ab $€ 1200$ graduell und dann ab $€ 1240$ voll auf $65 \%$ abgesenkt. Die Kürzungen für Elterngeldbezieherinnen mit einem vorgeburtlichen Nettoeinkommen von über $€ 1200$ (d.h. unterhalb des durchschnittlichen Nettoeinkommens) betraf v. a. die (untere) „Mittelschicht“, die i.d.R. als ,,verdiente“ Gruppe konstruiert wird (Barbehön und Haus 2015).

3. Schließlich entfiel der Elterngeldanspruch für „Superreiche“ mit zu versteuerndem Einkommen von mehr als $€ 250.000$ (Singles) bzw. €500.000 (Verheiratete). Diese letzte Kürzungsmaßnahme betraf „Reichensteuer“-Pflichtige, wobei für „Superreiche“ regelmäßig Konstruktionen als Contenders beobachtbar sind.

\footnotetext{
${ }^{8}$ I.d.R. wurden ab 2007 zunächst $67 \%$ des entfallenden, vorherigen Einkommens bis zu einer Höhe von max. $1800 €$ ersetzt, wenn nach der Geburt eines Kindes keine Erwerbstätigkeit oder eine Erwerbstätigkeit von max. 30 Wochenstunden ausgeführt wird.

${ }^{9}$ Das Elterngeld kann daher als Mischform aus erwerbsbasierter und bürgerschaftsbasierter Leistung eingestuft werden (Blum und Dobrotić 2018).
} 
In der Corona-Pandemie wurden zwei Elterngeldreformen vorgenommen. Im Mai 2020 wurden drei spezifische Pandemiemaßnahmen beschlossen: 1) Beschäftigte in der „kritischen Infrastruktur“ durften ihren Elterngeldbezug über den (regulär möglichen) Bezugszeitraum hinaus aufschieben; 2) der Partnerschaftsbonus entfällt auch dann nicht, wenn Eltern pandemiebedingt mehr oder weniger arbeiten als geplant; 3) während der Pandemie gezahlte Einkommensersatzleistungen (z. B. Kurzarbeitergeld) reduzieren nicht die Höhe des Elterngelds von werdenden Eltern, sondern werden bei der Elterngeldberechnung ausgeklammert (BMFSFJ 2020). Mit Beschluss vom 29.01.2021 wurden verschiedene Flexibilisierungen (z.B. Erhöhung der zulässigen Teilzeitarbeit bei Elterngeldbezug und Elternzeit von 30 auf 32 Wochenstunden) und einzelne Leistungsverbesserungen (zusätzlicher „Frühchen-Monat“) vorgenommen, aber auch eine Kürzungsmaßnahme durchgeführt, nämlich die in der 2010er Reform erfolgte Höchsteinkommensgrenze für einen Elterngeldbezug für „Spitzenverdienerinnen“ weiter abgesenkt auf nun 300.000€ für Paare (für Alleinerziehende weiterhin 250.000€). Mit Blick auf Zielgruppen standen in den 2020/21 durchgeführten Reformen die unmittelbaren Krisenreaktionen im Sinne einer ,sozialpolitischen Unterstützung“ im Vordergrund; es ging v. a. um Flexibilisierungen und Abfederung der Krisenfolgen, z. B. für von Kurzarbeit betroffene werdende Eltern.

\subsubsection{Krisennarrative zum Elterngeld}

Finanzkrise Angesichts von Verschuldung, Stabilitätspakt und der nun im Grundgesetz festgelegten Schuldenbremse - so Finanzminister Schäuble (CDU) vor einer Regierungsklausur zum Sparpaket im Juni 2010 - werde das Jahr 2011 einen ,haushaltspolitischen Wendepunkt" bringen und ,,allen Beteiligten müsse klar sein, dass kein Politikbereich ausgenommen werden könne“ (Deutsche Welle, 14.01.2010). Auch Familienministerin Schröder (CDU) bezog sich argumentativ auf den Sparzwang und kündigte bereits vorab einen eigenen Beitrag zum „Spargebot“ (SZ 2010a) für den Bereich des Elterngelds an. Trotz dieses Settings wurden von Regierungsseite nicht primär taking-out-of-helplessness-Narrative zur Reformlegitimation erzählt, wenngleich Sparzwang-Argumente im weiteren Verlauf immer wieder genutzt wurden. Die zentralen Fragen drehten sich jedoch darum, in welchen Bereichen ${ }^{10}$ und bei wem gekürzt werden sollte und wie die Lasten des Sparpakets im Allgemeinen sowie des Elterngelds im Speziellen zwischen verschiedenen sozialen Gruppen verteilt wurden.

Zunächst stand die Streichung des Elterngelds für Hartz-IV-Empfänger im Fokus. Zwar wurde die Kürzung von Merkel (FAZ 2010a) und Schröder (SZ 2010a) als „schmerzliche Entscheidung“ beschrieben. Sie wurde also z.T. verknüpft mit einem allgemeinen taking-to-control-Narrativ, das die Richtigkeit des Sparens zur Wiedererlangung von Kontrolle betonte. ${ }^{11}$ Primär wurden für die Elterngeldstrei-

\footnotetext{
10 Merkel erklärte bereits vorab die Bildungsausgaben sowie die Renten ,für tabu“ (SZ 2010a) im Hinblick auf Kürzungen, also sowohl einen zentralen Social-investment-Bereich sowie einen klassischen OSRBereich.

11 So z. B. Merkel: „Es sind ernste Zeiten. Es sind schwierige Zeiten. Wir können uns nicht all das, was wir uns wünschen, leisten, wenn wir die Zukunft gestalten wollen“ (Spiegel online 2010).
} 
chung Argumente genutzt, die eine „Sachlogik“ in den Vordergrund stellten und somit auch als Versuch gelesen werden können, die Entscheidung zu rationalisieren. Ein Aspekt hierbei war die ordnungspolitische Logik: Auch das Kindergeld werde auf die Grundsicherungsleistungen angerechnet, so Schröder, die zusätzliche Zahlung des Elterngelds sei daher von Beginn an ,systemwidrig“ gewesen (SZ 2010b). Diese Logik wird mit dem Lohnabstandsgebot und der Notwendigkeit von Arbeitsanreizen auch als richtig begründet: Ein zusätzliches Elterngeld könne für Hartz-IVEmpfängerinnen ,,auch eine negative Wirkung entfalten“, so Schröder, ,,wer arbeiten gehe, müsse mehr haben als derjenige, der nicht arbeite“ (FAZ 2010d). Für die geringverdienenden „Aufstocker“ sowie Minijobber, denen zunächst ebenfalls das Elterngeld gestrichen werden sollte, wurde nach Protest eine Ausnahmeregelung vorgesehen, um, so Schröder, ,diejenigen nicht zu bestrafen, die versuchen, durch Erwerbsarbeit ihren Lebensunterhalt aus eigener Kraft zu sichern“ (FAZ 2010b). Insinuiert scheint hier durchaus, dass nicht-arbeitende Hartz-IV-Empfänger eben nicht versuchten, ,ihren Lebensunterhalt aus eigener Kraft zu sichern“, d.h. sie werden zumindest implizit als Deviants beschrieben. So auch der stellvertretende Fraktionsvorsitzende Fuchs (CDU):

Ich halte es für richtig, dass wir in bestimmten Bereichen Einsparungen vorgenommen haben. [...] Wenn wir beim Elterngeld Einsparungen vorgenommen haben, dann haben wir das deswegen getan, weil das Elterngeld bei Hartz-IVFamilien falsch angesetzt ist. [...] Wenn heute eine Hartz-IV-Familie mit zwei Kindern inklusive Elterngeld rund $1870 €$ netto erhält, dann führt das dazu, dass sich sehr viele dem ersten Arbeitsmarkt nur relativ zögerlich zur Verfügung stellen. Das muss korrigiert werden, und das wollen wir tun (Deutscher Bundestag 2010, S. 5253).

Die Kürzung sei „schmerzhaft aber gerecht“ (FAZ 2010b), so das Ministerium. Insofern ist für die Streichung des Elterngelds für Langzeitarbeitslose eine takingto-take-Erzählung zu diagnostizieren, welche die „Richtigkeit“ des Nehmens über Sachlogiken und z. T. auch deviantes Verhalten der Zielgruppe konstruiert.

Die Reduzierung der Einkommensersatzrate von $67 \%$ auf $65 \%$ erhielt in der politischen Diskussion und auch Legitimierung der Reform vergleichsweise wenig Aufmerksamkeit. Selbst in den Medien bewertete z. B. die SZ (2010b), dass diese „Einschränkung [...] für Gutverdiener verkraftbar sein dürfte, zumal in den letzten Tagen deutlich drastischere Einbußen im Gespräch waren“. Merkel argumentierte z.B., man habe nicht nur bei „,den Unteren“ (Hartz IV) gekürzt, sondern hiermit auch bei „den Oberen“ (SZ 2010c) - hiervon lässt sich allerdings bei einem Nettoeinkommen von $€ 1240$ kaum sprechen, de facto griff die Kürzungsmaßnahme ab einem Nettoeinkommen von $€ 2770$ gar nicht mehr, da ab dieser Summe der Elterngeld-Höchstbetrag von $€ 1800$ erreicht war. Im Hinblick auf das Narrativ zur Kürzung der Einkommensersatzrate kann jedoch aus unserem Material kein eindeutiges Reformnarrativ (jenseits der allgemeinen Einbettung in taking-to-control) abgeleitet werden.

Die FDP brachte über ihre Familiensprecherin Gruß wiederholte Kürzungsvorschläge für zusätzliche Gruppen ein, die alle in Richtung einer höheren ,,sozialen Gerechtigkeit“ abzielten. Dies muss auch vor dem Hintergrund der damaligen Um- 
fragewerte der FDP (die ab 2013 nicht mehr im Bundestag vertreten war) gesehen werden, der insbesondere die sogenannten „Hoteliersteuer“ kurz zuvor scharfe Kritik einer Lobbypolitik eingebracht hatte. So schlug die FDP z. B. vor, das Elterngeld für alle vor der Geburt nicht Erwerbstätigen zu streichen (FAZ 2010c), der Logik einer Einkommensersatzleistung folgend. Aus „Gerechtigkeitsgründen“ solle das Elterngeld nicht nur für Hartz-IV-Empfänger, sondern auch für nichtberufstätige ,Millionärsgattinnen“ entfallen, nutzte Gruß eine entsprechende Synekdoche (SZ 2010d). Hier werden Gruppen gegenübergestellt und im Zuge einer Contender-Konstruktion ein taking-to-take-Narrativ entwickelt. Der FDP-Vorstoß zur Streichung des Mindestelterngelds wurde jedoch nicht weiterverfolgt. Wie oben beschrieben, kann die Hauptzielgruppe des Mindestelterngelds als Betreuungsleistung, nämlich die „Hausfrauen“ im traditionellen ,männlichen Ernährermodell“, als Advantaged eingeordnet werden.

Als - letztlich umgesetzten - Vorschlag brachte die FDP dann ein, das Elterngeld für Personen zu streichen, die Reichensteuer zahlen: Die Kürzungen beim Elterngeld müssten „sozial ausgewogen gestaltet werden“, so Gruß, und „,wer Reichensteuer zahlt, ist auf das Elterngeld nicht angewiesen“ (SZ 2010e). Als Parlamentarischer Geschäftsführer der Unionsfraktion bewertete Altmaier (CDU) diesen Vorschlag als ,,vertretbar und begründbar“ (FAZ 2010d). Gemessen an den durch diese Kürzungsmaßnahme real erwarteten begrenzten Einsparungen ${ }^{12}$ kann ihr sicherlich ein überdurchschnittlicher Symbolcharakter attestiert werden, der von der FDP auch strategisch als Widerlegung der „Klientelpolitik für Superreiche“ genutzt werden sollte. Konstruiert wurden die „Superreichen“ zwar nicht im negativen Sinn als Contenders, aber doch insofern ,unverdient“ im Zuge eines taking-to-take-Narratives, als dass sie die Leistung nicht benötigten und ihren Teil der Verantwortung übernehmen sollten, damit es beim (notwendigen) Sparen ,gerecht“ zugehe.

Coronakrise Insgesamt zeigt sich für die Corona-Krise, dass die Kita- und Schulschließungen im Zuge der Pandemiebekämpfung sowie deren Folgen für Eltern und Kinder das familienpolitische Feld dominierten (darunter auch Forderungen nach einem „Corona-Elterngeld“ ${ }^{13}$, die insbesondere seitens der Grünen eingebracht wurden). Den Elterngeld-Änderungen wurde demgegenüber keine große Aufmerksamkeit geschenkt und die Diskussionen darum erreichten längst nicht das Ausmaß der 2010 im Rahmen des Sparpakets beschlossenen Änderungen - was (neben dem dominanten Fokus auf den Kita- und Schulschließungen) auch damit zusammenhängt, dass 2020/21 eher selektive Expansionsmaßnahmen verhandelt wurden, während der Retrenchment-Charakter gegenüber 2010 begrenzt blieb.

Die Beschlüsse im Mai 2020 stellten spezifische Pandemiemaßnahmen dar, die allerdings unterschiedliche Gruppen von Eltern betrafen. Mit dem Ausgleich möglicher Folgen insbesondere von Kurzarbeit standen aktuelle Elterngeld-Bezieherinnen

\footnotetext{
12 Hiermit sollten jährlich 3,8 Mio. $€$ gespart werden (ca. 2200 Betroffene) - gegenüber einer Ersparnis von knapp 350 Mio. $€$ bei den Langzeitarbeitslosen (SZ 2010e).

13 Zur Einführung eines Corona-Elterngeldes kam es nicht. Die Kita- und Schulschließungen im Frühjahr 2020 sollten v. a. über Lohnfortzahlungen auf Basis des Infektionsschutzgesetzes aufgefangen werden; bei den (Teil-)schließungen im Winter 2020/21 kamen zusätzliche Kinderkrankentage hinzu.
} 
(Partnerschaftsbonus) bzw. zukünftige Elterngeld-Bezieher (Elterngeldberechnung) als Zielgruppen im Mittelpunkt, denen narrativ Unterstützung in der Krise zugesichert wurde:

Trotz Corona-Krise müssen sich Eltern und die, die es demnächst werden, keine Sorgen ums Elterngeld machen. Es ist krisenfest. Mit der Elterngeld-Reform senden wir ein klares Signal: Auch in der Corona-Krise können sich Mütter und Väter auf Deutschlands bekannteste und beliebteste Familienleistung verlassen (BMFSFJ 2020).

Deutschlands „bekannteste und beliebteste Familienleistung“ werde „krisenfest“ gemacht, so Familienministerin Giffey (SPD) im Zuge eines giving-to-give-Narrativs (Deutscher Bundestag 2020b, S. 19235) (vgl. auch Blum und Kuhlmann 2021). Eltern changieren hier über ihre Adressierung als von Kurzarbeit betroffene Erwerbstätige gewissermaßen zwischen (zu stärkenden) Dependents und (gefährdeten) Advantaged. Die dritte vorgenommene Änderung, die ,systemrelevanten Beschäftigten“ ermöglichte, den Elterngeldbezug über den (regulär möglichen) Zeitraum hinaus aufzuschieben, wurde narrativ eher in ein giving-to-promote gekleidet. Das Narrativ konzentriert sich in diesem Sinne auf die Befähigung dieser Gruppe: Befähigung zum einen, um aktuell eben keine Elternzeit zu nehmen, sondern während der Pandemie in der „kritischen Infrastruktur“ bereitzustehen, aber dadurch auch die Möglichkeit zu geben, „die Zeit mit ihren Kindern nach der Krise nachzuholen“ (BMFSFJ 2020; SZ 2020b). So auch Giffey bei ihrer Rede im Bundestag:

Wir kümmern uns um Eltern in systemrelevanten Berufen, zum Beispiel in Krankenhäusern und in Altenheimen, die gerade gebraucht werden und deshalb ihre Elternzeit nicht wie geplant antreten können (Deutscher Bundestag 2020b, S. 19235).

Die im Januar 2021 beschlossenen Änderungen hingegen standen nicht in einem unmittelbaren Krisenzusammenhang, da sie großteils bereits Mitte Februar 2020 und damit vor dem Beginn der ersten Pandemiewelle in Deutschland auf die Agenda gesetzt, im September 2020 vom Bundeskabinett auf den Weg gebracht und schließlich im Januar 2021 beschlossen wurden. Die Expansionsmaßnahmen sollen zum einen den Partnerschaftsbonus attraktiver machen, der ,die Beteiligung von Vätern nachweislich [stärke]“ (SZ 2020c), zum anderen solle durch eine längere Zahlung des Elterngeldes im Fall von Frühchen den Eltern mehr Zeit gegeben werden, ,um mögliche Entwicklungsverzögerungen ihres Kindes besser auffangen zu können“ (ebd.). So auch Giffey in der Parlamentsdebatte:

Das alles dient dazu, starke Familien in einem starken Land zu unterstützen, dazu, dass Frauen wie Männer Kinder haben können, sich um die Familie kümmern können, aber auch im Beruf erfolgreich sein können. [...] Und das ist wichtig für eine moderne Gesellschaft und für ein zukunftsfähiges Land (Deutscher Bundestag 2020g, S. 24722).

Im Sinne eines giving-to-promote-Narratives wird hier der Zweck der sozialpolitischen Leistungen als Befähigung zu zukünftigem Handeln in den Vordergrund gerückt. Die - zur Finanzierung dieser Expansionsmaßnahmen dienende - Leis- 
tungskürzung für Spitzenverdiener hingegen schließt an die Sparmaßnahme aus dem Jahr 2010 an, da die damals festgelegte Höchsteinkommensgrenze von 500.000€ auf nunmehr $300.000 €$ weiter abgesenkt wurde. Ähnlich wie 2010 nahm die Legitimierung der Kürzung den Charakter eines taking-to-take an, so z. B. im Gesetzentwurf:

Bei einem derart hohen Einkommen ist davon auszugehen, dass Elterngeld für die Entscheidung, in welchem Umfang zugunsten der Betreuung des Kindes auf Erwerbstätigkeit verzichtet werden soll, unerheblich ist, Vor diesem Hintergrund ist eine Herabsetzung des Grenzbetrags für Paare mit einem gemeinsamen Elterngeldanspruch angemessen (FAZ 2020).

Als Eltern „mit einem derart hohen Einkommen“ scheinen die „Spitzenverdiener" zumindest tendenziell als (privilegierte) Contenders in den Blick zu rücken, die zwar nicht negativ, aber doch in dem Sinne ,unverdient“" konstruiert werden, dass kein Bedarf bestehe, sie sozialpolitisch zu unterstützen: „Für sie ist die eigenständige Vorsorge für den Zeitraum der Elternzeit auch ohne Elterngeld möglich“ (BMFSFJ 2021). Dass diese Änderung z. B. bei der parlamentarischen Debatte zur Änderung gar nicht thematisiert wurde, demonstriert, dass kein Policy-Akteur die i.d.S. „Unverdientheit“ der Gruppe in Frage stellte und dass Kürzungsmaßnahmen nicht notwendigerweise narrativ legitimiert werden müssen (vgl. Görres et al. 2019).

\subsection{Politische Maßnahmen und narrative Strategien im Vergleich}

Das Setting zwischen den Kurzarbeitsreformen in der Finanz- und Coronakrise unterschied sich weniger stark als das der Elterngeldreformen, denn in beiden Fällen ging es - im Zuge sozialstaatlichen Ausbaus - darum, mit der Kurzarbeit eine „Brücke“ über das „wirtschaftliche Tal“ zu bauen (Deutscher Bundestag 2020d, S. 19712). Dementsprechend werden in beiden Kontexten (teilweise miteinander verknüpft) giving-to-give- und giving-to-promote-Narrative sichtbar. Auf Ebene der individuellen Arbeitnehmerinnen wird die verdiente sozialpolitische Unterstützung hervorgehoben; zugleich wird mit Blick auf Betriebe und die gesamtwirtschaftliche Lage in beiden Krisen der Aspekt der „Befähigung“ hervorgehoben. Betrachtet man allerdings die in den Narrativen konstruierten Arbeitnehmerinnen als Zielgruppen genauer, treten Unterschiede zwischen beiden Krisenkontexten hervor. In der Finanzkrise bilden (tendenziell gutverdienende) Arbeitsmarktinsider (z.B. aus der Automobilindustrie) und somit Advantaged die Hauptzielgruppe der giving-to-give-Narrative; auch wenn z.T. Leiharbeiterinnen als eher Arbeitsmarktoutsider und somit Dependents hinzutreten, welche - anders als in o.g. Typologie konzeptualisiert - ebenfalls in giving-to-give-Narrativen als Zielgruppe konstruiert werden. In der Coronakrise werden entsprechend der viel umfassenderen Nutzung von Kurzarbeit und der starken Betroffenheit vieler Branchen mit häufig niedrigen Löhnen (z.B. Gastronomie) neben den Advantaged in stärkerem Maße auch Dependents in giving-to-give-Narativen konstruiert. Dementsprechend finden sich z. B. verstärkt Narrative zur Aufstockung des Kurzarbeitergelds, in denen Arbeitnehmerinnen nicht ausreichend verdienen, um mit dem regulären Kurzarbeitergeld über die Runden zu kommen. War Weiterqualifizierung als von giving-to-promote-Narrativen fokussier- 
te „Lösung“ in der Finanzkrise sehr wichtig, kommt sie in der Coronakrise erst später prominent auf die Agenda. Diese bezieht sich ausdrücklich nicht nur auf Dependents, sondern auch auf Advantaged.

Die empirischen Befunde mit Blick auf die sozialpolitischen Expansionsmaßnahmen entsprechen somit nur bedingt der Typologie sozialpolitischer Reformnarrative (vgl. Tab. 1): Denn giving-to-give-Narrative werden hier sowohl für Advantaged-als auch für Dependents-Gruppen eingesetzt, zugleich finden sich auch für Advantaged hier giving-to-promote-Erzählungen. Ein Grund hierfür könnte sein, dass sich in Krisenkontexten Gruppenkonstruktionen verschieben können (vgl. auch Blum und Kuhlmann 2021). Eine andere These wäre, dass sich sozialpolitische Gruppen zunehmend weniger klar abgrenzbar darstellen als in der Vergangenheit, z. B. aufgrund unsicherer Lebens- und Erwerbsverläufe, vor denen auch Gruppen, die ansonsten als Advantaged charakterisiert werden, nicht gefeit sind (vgl. auch Häusermann et al. 2015).

Bei Sparpaket und Elterngeldkürzungen 2010 dominierten Fragen der Lastenverteilung zwischen sozialen Gruppen. Während die Regierung das Sparpaket als ,ausgewogen und gerecht“ bezeichnete und Merkels erklärtes Ziel bei den Einsparungen die „soziale Gerechtigkeit“ (SZ 2010a) war, wurde es z.B. von SPDFraktionschef Steinmeier als „Umverteilung von unten nach oben“ (SZ 2010a) bewertet. Dementsprechend stand die Verdientheit verschiedener Gruppen im Mittelpunkt der Elterngeldreform 2010. Durch den Kontext des Sparpakets und die damit verbundene Geschichte eines taking-to-control war der Rahmen gesetzt, innerhalb dessen konkretere Geschichten des Nehmens erzählt und verhandelt wurden. Dass bei den Kürzungen für die Gruppe der Hartz-IV-Empfänger sowie der Reichensteuer-Pflichtigen auch taking-to-take-Argumentationen sichtbar wurden, entspricht der sozialen Konstruktion dieser Gruppen im politischen Prozess als ,unverdiente“ Deviants und Contenders (Schneider und Ingram 1993, Blum und Kuhlmann 2019). Für die „Mittelschicht“-Gruppe der Elterngeldbezieherinnen, die Einschnitte bei der Ersatzrate hinnehmen musste, fand sich allerdings neben einer gewissen Relativierung (,leichte Einbußen“ für „,höhere Einkommen“) v. a. eine Nicht-Thematisierung. Der Krisenkontext der Elterngeldreformen in der Corona-Pandemie bot ein anderes Setting: Hier stand (zumindest bis dato) kein Sparpaket aus ,ökonomischem Zwang“ im Mittelpunkt, sondern sozialpolitische Unterstützungsmaßnahmen durch Expansion bzw. großzügigere Regelungen. Häufig wiederholt wurde das Bild, Deutschlands „beliebteste Familienleistung krisenfest“ zu machen (BMFSFJ 2020), was als givingto-give verstanden werden kann. Insbesondere mit Blick auf die systemrelevanten Gruppen stand aber auch i.S.v. giving-to-promote eine Befähigung im Fokus - und zwar sowohl als Arbeitnehmerinnen als auch (im Anschluss) als Eltern. Mit der weiteren Einschränkung des Elterngeldbezugs für Spitzenverdienerinnen wird latent ein taking-to-take-Narrativ sichtbar, das aber die „Unverdientheit“ dieser Gruppe als so selbstverständlich setzte, dass es beinahe ebenfalls einer narrativlosen Leerstelle glich. 


\section{Fazit}

Narrative sind ein zentrales Element, um Ungleichheiten, die durch sozialpolitische Reformen geschaffen oder ausgeglichen werden, im politischen Prozess zu legitimieren. Für die untersuchten Maßnahmen in Krisenzeiten - das Kurzarbeiter- und das Elterngeld - konnte in diesem Artikel gezeigt werden, dass Narrative und die in ihnen enthaltenen Zielgruppenkonstruktionen ein zentrales Element in der Reformkommunikation darstellen. In Narrativen beschreiben Erzählerinnen sozialpolitische Reformen und Reformkontexte und stellen diese in einen Sinnzusammenhang, der Legitimationszwecken dient. Erzählungen über ,faire“ Reformen können dabei auch materielle Kürzungen umdeuten oder überdecken. In der Tat sollte der Fokus dieses Beitrags auf Legitimationsstrategien sozialpolitischer Maßnahmen nicht darüber hinwegtäuschen, dass Regierungsakteure auch auf sozialpolitische Narrative - und die Konstruktion von Gruppen als „,verdient“ - rekurrieren können, ohne dass dies sozialpolitische Reformen zur Folge hat. Im Zuge der Corona-Pandemie kann dies beispielsweise in Bezug auf in Pflegeberufen tätige Menschen festgestellt werden, deren „Systemrelevanz“ weithin geteilt wurde. Verbunden wurde diese Anerkennung mit der Forderung, dass diese „Helden des Alltags“ „,nicht nur warme Worte, sondern langfristig auch bessere Löhne“ (BMAS 2020) verdienten - was jedoch von staatlicher Seite allein nicht entschieden werden könne (und bis heute trotz Pflegereform nicht in nennenswerter Weise erfolgt ist). In einem ähnlichen Sinne konstatiert Smith Ochoa (2020, S. 320): ,(The) discrepancy between growing outcry about inequality and the relative policy standstill is puzzling and challenges political science to analyze this missing change."

Inwieweit die Befunde dieses Beitrags auch auf andere Fälle übertragbar sind, muss weiterführende empirische Forschung zeigen, die neben unterschiedlichen Politikfeldern auch unterschiedliche Reformkontexte einbeziehen sollte. Darüber hinaus erscheint uns für die Zukunft ein vertiefter Blick auf narrative Strategien der Nicht-Thematisierung durch „Narrativlosigkeit“ bedeutsam, der sich in diesem Beitrag nur andeutete. Gemeint ist damit eine gerade nicht stattfindende Vermittlung und Legitimation von sozialpolitischem Rückbau (oder fehlendem Ausbau) in Bezug auf (bestimmte) von der Reform betroffene Zielgruppen. Bei der Elterngeldreform 2010 fand sich z. B. ein weitreichendes „Nicht-Narrativ“ in Bezug auf die Kürzungen für die Mittelschicht. ${ }^{14}$ Tatsächlich kann es für Politikerinnen durchaus attraktiv sein, negative Aspekte politischen Handelns nicht zu thematisieren (vgl. auch Görres et al. 2019). Somit können Narrative auch als Leerstelle im politischen Reformprozess dem Verständnis (sozial-)politischer Reformen eine neue, bislang wenig beachtete Facette hinzufügen.

Danksagung Wir bedanken uns bei Christopher Smith Ochoa und Taylan Yildiz für die Organisation des Special Issues zu Narrativen sozioökonomischer Ungleichheit. Marlon Barbehön und den anonymen Gutachter*innen gilt unser Dank für sehr hilfreiche Kommentare zu einer früheren Version dieses Beitrags.

Förderung Die Forschung von Johanna Kuhlmann wurde gefördert durch die Deutsche Forschungsgemeinschaft (DFG) - Projektnummer 374666841 - SFB 1342.

14 Auf Basis theoretischer Überlegungen hätte erwartet werden können, dass der Rückbau der populären Maßnahme für diese ,,verdiente“ Gruppe unter Rückgriff auf die eigene Hilflosigkeit legitimiert würde. 
Funding Open Access funding enabled and organized by Projekt DEAL.

Open Access Dieser Artikel wird unter der Creative Commons Namensnennung 4.0 International Lizenz veröffentlicht, welche die Nutzung, Vervielfältigung, Bearbeitung, Verbreitung und Wiedergabe in jeglichem Medium und Format erlaubt, sofern Sie den/die ursprünglichen Autor(en) und die Quelle ordnungsgemäß nennen, einen Link zur Creative Commons Lizenz beifügen und angeben, ob Änderungen vorgenommen wurden.

Die in diesem Artikel enthaltenen Bilder und sonstiges Drittmaterial unterliegen ebenfalls der genannten Creative Commons Lizenz, sofern sich aus der Abbildungslegende nichts anderes ergibt. Sofern das betreffende Material nicht unter der genannten Creative Commons Lizenz steht und die betreffende Handlung nicht nach gesetzlichen Vorschriften erlaubt ist, ist für die oben aufgeführten Weiterverwendungen des Materials die Einwilligung des jeweiligen Rechteinhabers einzuholen.

Weitere Details zur Lizenz entnehmen Sie bitte der Lizenzinformation auf http://creativecommons.org/ licenses/by/4.0/deed.de.

\section{Literatur}

Bäcker, Gerhard, Gerhard Naegele, und Reinhard Bispinck (Hrsg.). 2020. Sozialpolitik und soziale Lage in Deutschland. Ein Handbuch, 6. Aufl., Wiesbaden: Springer VS.

Barbehön, Marlon, und Michael Haus. 2015. Middle class and welfare state-Discursive relations. Critical Policy Studies 9:473-484. https://doi.org/10.1080/19460171.2015.1009840.

Blank, Florian. 2020. Sozialpolitik in der Corona-Krise. WSI-Blog. https://www.wsi.de/de/blog-1785723723.htm. Zugegriffen: 17. Febr. 2021.

Blum, Sonja. 2019. Reform narratives and argumentative coupling in German pension policy: constructing the 'deserving retiree'. Policy and Society 38:389-407. https://doi.org/10.1080/14494035.2019. 1655130.

Blum, Sonja, und Ivana Dobrotić. 2018. Wer hat Zugang zu Elterngeld? Soziale Rechte und Anspruchsbedingungen in vergleichender Perspektive. Sozialer Fortschritt 67(8/9):667-687. https://doi.org/10. 3790/sfo.67.8-9.667.

Blum, Sonja, und Johanna Kuhlmann. 2019. Stories of how to give or take-Towards a typology of social policy reform narratives. Policy and Society 38:339-355. https://doi.org/10.1080/14494035.2019. 1657607.

Blum, Sonja, und Johanna Kuhlmann. 2021. Wir sind Helden. Sozialpolitische Maßnahmen und politische Kommunikation in der Corona-Pandemie. dms - der moderne staat-Zeitschrift für Public Policy, Recht und Management 14:411-432. https://doi.org/10.3224/dms.v14i2.04.

BMAS. 2020. „Soziale Marktwirtschaft ist das beste Mittel“. Interview von Hubertus Heil, Bundesminister für Arbeit und Soziales, mit der Funke Mediengruppe. 26.03.2020. https://www.bmas.de/DE/Service/ Presse/Interviews/2020/2020-03-26-funke-mediengruppe.html. Zugegriffen: 26. Febr. 2021.

BMAS. 2021. Erleichtertes Kurzarbeitergeld. https://www.bmas.de/DE/Corona/erleichtertes-kurzarbeiter geld.html. Zugegriffen: 22. Dez. 2021.

BMFSFJ. 2020. Reform des Elterngeldes. Neue Regelungen stützen Familien in der Corona-Krise. Pressemeldung, 15.05.2020. https://www.bmfsfj.de/bmfsfj/aktuelles/alle-meldungen/elterngeld-wirdkurzfristig-angepasst-154564?view=. Zugegriffen: 27. Febr. 2021.

BMFSFJ. 2021. Verbesserungen im Elterngeld treten in Kraft. Pressemeldung, 31.08.2021. https://www. bmfsfj.de/bmfsfj/aktuelles/alle-meldungen/elterngeld-verbesserungen-gesetz-aenderung- 160558. Zugegriffen: 3. Jan. 2022.

Boin, Arjen, Paul 't Hart, Eric Stern, und Bengt Sundelius. 2016. The politics of crisis management. Public leadership under pressure, 3. Aufl., Cambridge: Cambridge University Press.

Bonoli, Giuliano. 2005. The politics of the new social policies: Providing coverage against new social risks in mature welfare states. Policy \& Politics 33:431-449. https://doi.org/10.1332/0305573054325765.

Cox, Robert Henry. 2001. The social construction of an imperative: Why welfare reform happened in Denmark and the Netherlands but not in Germany. World Politics 53:463-498.

Deutscher Bundestag (2008a): Stenografischer Bericht der 185. Sitzung vom 4. November 2008 (=BTPlenarprotokoll 16/185). 
Deutscher Bundestag (2008b): Stenografischer Bericht der 186. Sitzung vom 12. November 2008 (=BTPlenarprotokoll 16/186).

Deutscher Bundestag (2008c): Stenografischer Bericht der 189. Sitzung vom 26. November 2008 (=BTPlenarprotokoll 16/189).

Deutscher Bundestag (2008d): Stenografischer Bericht der 190. Sitzung vom 27. November 2008 (=BTPlenarprotokoll 16/190).

Deutscher Bundestag (2009a): Stenografischer Bericht der 198. Sitzung vom 14. Januar 2009 (=BT-Plenarprotokoll 16/198).

Deutscher Bundestag (2009b): Stenografischer Bericht der 203. Sitzung vom 30. Januar 2009 (=BT-Plenarprotokoll 16/203).

Deutscher Bundestag (2010): Stenografischer Bericht der 51. Sitzung vom 1. Juli 2010 (=BT-Plenarprotokoll 17/51).

Deutscher Bundestag (2020a): Stenografischer Bericht der 154. Sitzung vom 25. März 2020 (=BT-Plenarprotokoll 19/154).

Deutscher Bundestag (2020b): Stenografischer Bericht der 155. Sitzung vom 22. April 2020 (=BT-Plenarprotokoll 19/155).

Deutscher Bundestag (2020c): Stenografischer Bericht der 156. Sitzung vom 23. April 2020 (=BT-Plenarprotokoll 19/156).

Deutscher Bundestag (2020d): Stenografischer Bericht der 159. Sitzung vom 13. Mai 2020 (=BT-Plenarprotokoll 19/159).

Deutscher Bundestag (2020e): Stenografischer Bericht der 164. Sitzung vom 29. Mai 2020 (=BT-Plenarprotokoll 19/164).

Deutscher Bundestag (2020f): Stenografischer Bericht der 193. Sitzung vom 20. November 2020 (=BTPlenarprotokoll 19/193).

Deutscher Bundestag (2020g): Stenografischer Bericht der 196. Sitzung vom 27. November 2020 (=BTPlenarprotokoll 19/196).

Deutsche Welle 2010. Schäuble schlägt Sparkurs ein. 14.01.2010. https://www.dw.com/de/schäubleschlägt-sparkurs-ein/a-5125366. Zugegriffen: 22. Dez. 2021.

Dodge, Jennifer. 2015. Indication and inference: reflections on the challenge of mixing paradigms in the Narrative Policy Framework. Critical Policy Studies 9:361-367. https://doi.org/10.1080/19460171. 2015.1075735 .

Dümig, Kathrin. 2010. Ruhe nach und vor dem Sturm: Die Arbeitsmarkt- und Beschäftigungspolitik der Großen Koalition. In Die zweite Große Koalition. Eine Bilanz der Regierung Merkel 2005-2009, Hrsg. Christoph Egle, Reimut Zohlnhöfer, 279-301. Wiesbaden: VS.

Esping-Andersen, Gøsta. 1990. The three worlds of welfare capitalism. Princeton: Princeton University Press.

Esping-Andersen, Gøsta, Duncan Gallie, Anton Hemerijck, und John Myles. 2002. Why we need a new welfare state. Oxford: Oxford University Press.

FAZ (faz.net). 2020. Mehr Geld für Eltern von Frühchen. 17.02.2020

FAZ. 2010a. Das größte Sparpaket in der Geschichte der Bundesrepublik, 08.06.2010., 1.

FAZ. 2010b. Breite Front gegen Ministerin Schröder, 17.07.2010., 4.

FAZ. 2010c. „Steuersenkung? Verzicht auf Erhöhung ist schon schwer!“, 20.08.2010., 4.

FAZ. 2010d. Kein Elterngeld für Spitzenverdiener, 27.10.2010., 13.

Fervers, Lukas. 2019. Economic miracle, political disaster? Political consequences of Hartz IV. Journal of European Social Policy 29:411-427. https://doi.org/10.1177/0958928718774259.

Fervers, Lukas, und Hanna Schwander. 2015. Are outsiders equally out everywhere? The eco-nomic disadvantage of outsiders in cross-national perspective. European Journal of Indus-trial Relations 21:369-387. https://doi.org/10.1177/0959680115573363.

Gadinger, Frank, Sebastian Jarzebski, und Taylan Yildiz. 2014. Politische Narrative. Konturen einer politikwissenschaftlichen Erzähltheorie. In Politische Narrative. Konzepte - Analysen - Forschungspraxis, 3-38. Wiesbaden: Springer VS.

Gehrke, Britta, und Enzo Weber. 2020. Kurzarbeit, Entlassungen, Neueinstellungen: Wie sich die CoronaKrise von der Finanzkrise 2009 unterscheidet. In: IAB-Forum 28.05.2020. https://www.iab-forum. de/kurzarbeit-entlassungen-neueinstellungen-wie-sich-die-corona-krise-von-der-finanzkrise-2009unterscheidet/. Zugegriffen: 16. Febr. 2021.

Gilbert, Neil, Harry Specht, und Paul Terrell. 1993. Dimensions of social welfare policy. Englewood Cliffs: Prentice Hall. 
Görres, Achim, Staffan Kumlin, und Rune Karlsen. 2019. Pressure without pain: What politicians (don't) tell you about welfare state change. Journal of Social Policy 48:861-884. https://doi.org/10.1017/ S0047279419000138.

Green-Pedersen, Christoffer. 2002. The politics of justification: Party competition and welfare state retrenchment in Denmark and the Netherlands from 1982 to 1998. Amsterdam: Amsterdam University Press.

Häusermann, Silja, Thomas Kurer, und Hanna Schwander. 2015. High-skilled outsiders? Labor market vulnerability, education and welfare state preferences. Socio-Economic Review 13:235-258. https:// doi.org/10.1093/ser/mwu026.

Hemerijck, Anton. 2015. The quiet paradigm revolution of social investment. Social Politics 22:242-256. https://doi.org/10.1093/sp/jxv009.

Husmann, Maria A. 2015. Social constructions of obesity target population: an empirical look at obesity policy narratives. Policy Sciences 48:415-442. https://doi.org/10.1007/s11077-015-9229-6.

Ingram, Helen M., und Anne L. Schneider. 2015. Making distinctions: The social construction of target populations. In Handbook of Critical Policy Studies, Hrsg. Frank Fischer, Douglas Torgerson, Anna Durnová, und Michael Orsini, 259-273. Cheltenham: Edward Elgar.

Kuhlmann, Johanna, und Sonja Blum. 2021. Narrative plots for regulatory, distributive, and redistributive policies. European Policy Analysis 7:276-302. https://doi.org/10.1002/epa2.1127.

Mätzke, Margitta. 2011. Political competition and unequal social rights. Journal of Public Policy 31:1-24. https://doi.org/10.1017/S0143814X1000022X.

Münch, Sybille. 2016. Interpretative Policy-Analyse: Eine Einführung. Wiesbaden: Springer VS.

Nygard, Mikael, und Nicole Krüger. 2012. Poverty, families and the investment state. The impact of social investment ideas on family policy discourses in Finland and Germany. European Societies 14:755-777. https://doi.org/10.1080/14616696.2012.726368.

OECD. 2013. Crisis squeezes income and puts pressure on inequality and poverty. http://www.oecd.org/ els/soc/OECD2013-Inequality-and-Poverty-8p.pdf. Zugegriffen: 23. Febr. 2021.

Pierson, Paul. 1994. Dismantling the welfare state? Reagan, Thatcher, and the politics of retrenchment. Cambridge: Cambridge University Press.

Sacchi, Stefano, Federico Pancaldi, und Claudia Arisi. 2011. The economic crisis as a trigger of convergence? Short-time work in Italy, Germany and Austria. Social Policy \& Administration 45:465-487. https://doi.org/10.1111/j.1467-9515.2011.00785.x.

Schneider, Anne, und Helen Ingram. 1993. Social construction of target populations: Implications for politics and policy. American Political Science Review 87:334-347. https://doi.org/10.2307/2939044.

Schulten, Thorsten, und Torsten Müller. 2020. Kurzarbeitergeld in der Corona-Krise. Aktuelle Regelungen in Deutschland und Europa. Policy Brief WSI 38, 04/2020. Düsseldorf: Hans-Böckler-Stiftung.

Schwander, Hanna, und Silja Häusermann. 2013. Who is in and who is out? A risk-based conceptualization of insiders and outsiders. Journal of European Social Policy 23:248-269. https://doi.org/10.1177/ 0958928713480064.

Smith Ochoa, Christopher. 2020. Trivializing inequality by narrating facts: a discourse analysis of contending storylines in Germany. Critical Policy Studies 14:319-338. https://doi.org/10.1080/19460171. 2019.1623056 .

Spiegel online. 2010. Koalition will 80 Milliarden Euro bis 2014 sparen, 07.06.2010. https://www.spiegel. de/politik/deutschland/kuerzungsplaene-von-union-und-fdp-koalition-will-80-milliarden-euro-bis2014-sparen-a-699111.html. Zugegriffen: 6. Nov. 2019.

Stone, Deborah. 2012. Policy paradox: The art of political decision making, 3. Aufl., New York: W.W. Norton.

SZ. 2009. Ein Geschenk für alle, 26.01.2009., 2.

SZ. 2010a. Das Elterngeld wird gekürzt, 02.06.2010., 6.

SZ. 2010b. Das Paket der Grausamkeiten, 08.06.2010., 2.

SZ. 2010c. Gestrichen voll, 08.06.2010., 3.

SZ. 2010d. Einwände aus allen Richtungen. 17.07.2010., 6.

SZ. 2010e. FDP will Elterngeld für Spitzenverdiener streichen, 11.10.2010., 1.

SZ. 2020a. Laden zu; Umsatz weg, 17.03.2020., 15.

SZ. 2020b. Coronavirus in Deutschland, 04./05.04.2020., 22.

SZ. 2020c. Mehr Elterngeld nach Frühgeburten geplant, 17.02.2020

Wenzelburger, Georg, und Felix Hörisch. 2016. Unpopular social policy reform and strategic communication: An introduction. Journal of Comparative Policy Analysis: Research and Practice 18:113-121. https://doi.org/10.1080/13876988.2015.1053262. 